\title{
Recurrent membranous tracheal stenosis with a diverticulum in a 9-year-old boy. Not all that wheezes is asthma
}

\author{
Frantisek Koprivaa ${ }^{a}$ Vitezslav Kolek ${ }^{b}$
}

Background. Benign tracheobronchial stenosis of the proximal airways may result from a variety of conditions and can cause dyspnea, cough, wheeze or stridor.

Methods and Results. In this report, we describe the case of a 9-year-old boy who was admitted to hospital with wheezing and progressive dyspnea lasting for six months. These abnormalities were diagnosed by multislice CT with 3-dimensional reconstruction of the trachea and by videobronchoscopy which demonstrated severe tracheal stenosis and an orifice of a tracheal diverticulum on the right side of the upper trachea. The stenosis was dilated with a balloon and vaporized with an Nd:YAG laser. Due to recurrent stenosis, the laser procedure had to be repeated several times in an approximately 4-6-month interval.

Conclusion. Association between recurrent membranous tracheal stenosis and a tracheal diverticulum is a rare medical condition.

Key words: membranous tracheal stenosis, diverticulum, laser

Received: October 10, 2015; Accepted: February 12, 2016; Available online: February 29, 2016

http://dx.doi.org/10.5507/bp.2016.009

${ }^{a}$ Department of Pediatrics, University Hospital Olomouc and Faculty of Medicine and Dentistry, Palacky University Olomouc, Czech Republic ${ }^{b}$ Department of Respiratory Medicine, University Hospital Olomouc and Faculty of Medicine and Dentistry, Palacky University Olomouc, Czech Republic

Corresponding author: Frantisek Kopriva, e-mail: frantisek.kopriva@fnol.cz

\section{INTRODUCTION}

Tracheal stenosis is a rare anomaly that occurs less often than laryngeal or other tracheal malformations. The narrowing results from complete or nearly complete rings of cartilage. Benign tracheobronchial stenosis of the proximal airways may result from a variety of conditions and can cause dyspnea, cough, wheeze, stridor or recurrent pulmonary infections. In infants, prolonged intubation, tracheotomy and congenital lesions are primary causes of stenosis and result most commonly in subglottic or tracheal lesions. Tracheal diverticula may be congenital or acquired. An association between recurrent membranous tracheal stenosis and a tracheal diverticulum is a rare medical condition.

In this report, we describe the case of a 9-year-old boy who was admitted to hospital with wheezing and progressive dyspnea lasting for six months. These abnormalities were diagnosed by multislice CT with 3-dimensional reconstruction of the trachea and by videobronchoscopy which demonstrated severe tracheal stenosis and an orifice of a tracheal diverticulum on the right side of the upper trachea. The stenosis was dilated with a $\mathrm{CRE}^{\mathrm{TM}}$ pulmonary balloon dilator smooth edge (Boston Scientific) and vaporized with an Nd:YAG laser. Due to recurrent stenosis, the laser procedure had to be repeated several times in approximately 4-6-month interval.

\section{CASE REPORT}

A 9-year-old boy was admitted to hospital with wheezing and progressive dyspnea lasting for six months and resulting in limited activities and some difficulty with reading. There was no past medical history of trauma to the respiratory tract or prior intubation. A computer tomography scan with three-dimensional reconstruction demonstrated stenosis of the trachea approximately 1.5 $\mathrm{cm}$ below the vocal cords and an orifice of a tracheal diverticulum $(6 \mathrm{~mm} \times 2 \mathrm{~mm})$ at the level of the second tracheal ring (Fig. 1). Flexible bronchoscopy showed circular stenosis of the trachea and an orifice of a tracheal diverticulum (Fig. 2). Pulmonary function tests demonstrated reduced flow rates, with no post-bronchodilator improvement (Fig. 3).

Vaporization of the diverticulum with an Nd:YAG laser of the TD (Sharplan 3000; $30 \mathrm{~W}$ energy) was carried out via rigid bronchoscopy under general anesthesia twice after a week. Balloon dilatation (Boston Scientific) was performed to increase in diameter of the trachea to $8 \mathrm{~mm}$. One week later, pulmonary function tests revealed normal parameters without pathological symptoms. Due to recurrent stenosis, however, the laser procedure had to be repeated several times in an approximately 4-6-month interval.

The study was approved by the Palacky University Hospital Ethics Committee. 


\section{DISCUSSION}

Tracheal stenosis is a rare anomaly that occurs less often than laryngeal or other tracheal malformations. The narrowing results from complete or nearly complete rings of cartilage. Three patterns are seen: (1) segmental stenosis that can occur anywhere in the tracheobronchial tree $(50 \%),(2)$ generalized or complete stenosis/hypoplasia $(30 \%)$ and (3) a funnel-shaped lesion often associated with a pulmonary artery sling $(20 \%)$. Tracheal stenosis is often associated with other malformations of the respiratory tract, esophagus or skeleton.

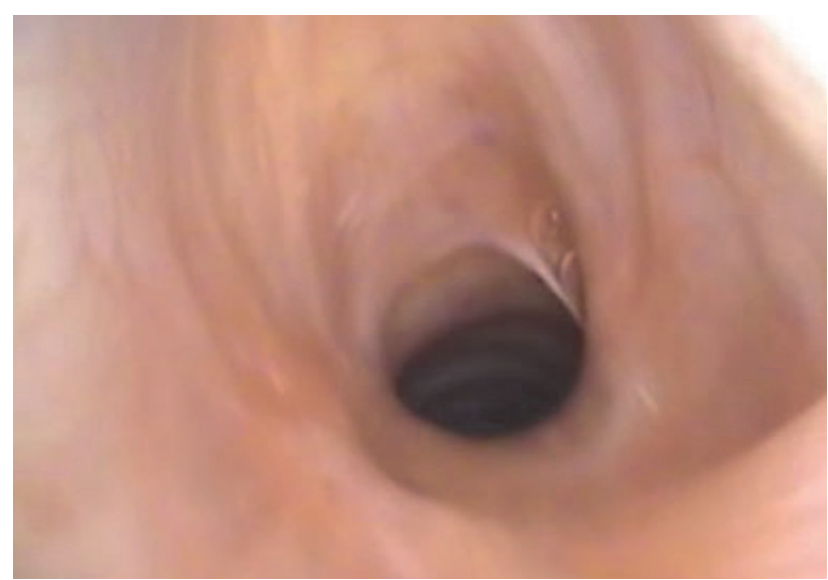

Fig. 1. Flexible videobronchoscopy shows membranous stenosis of the trachea.
Benign tracheobronchial stenosis of the proximal airways may result from a variety of conditions and can cause dyspnea, cough, wheeze, stridor or recurrent pulmonary infections. Acquired subglottic stenosis is related to a variety of causes, including prolonged endotracheal intubation, gastroesophageal reflux, infection, autoimmune disorders and iatrogenic disorders.

Conditions potentially causing strictures may also ultimately result in fibrotic scarring of the airways; however, there may be other initiating mechanisms. For example, fibrotic anastomotic airway stenosis may occur in lung transplant recipients from either airway rejection or ischemia.

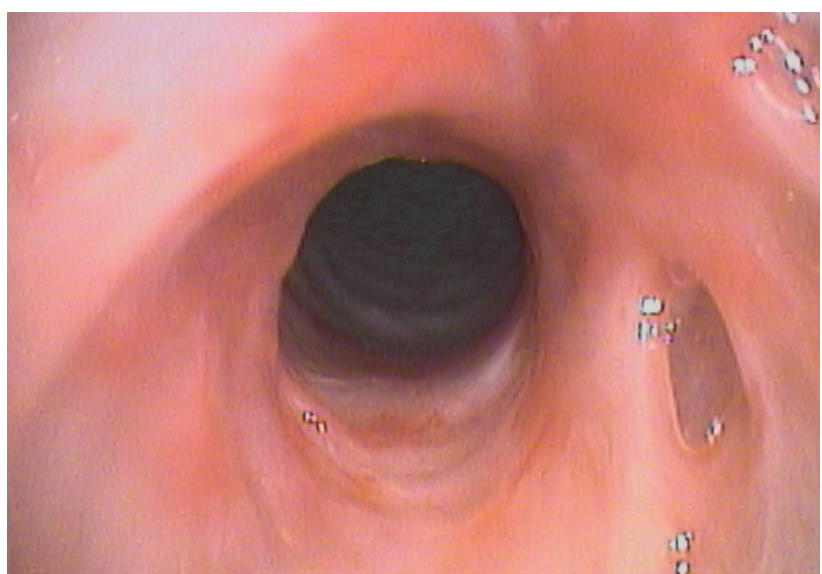

Fig. 2. Flexible videobronchoscopy shows membranous stenosis of the trachea and an orifice of a tracheal diverticulum.

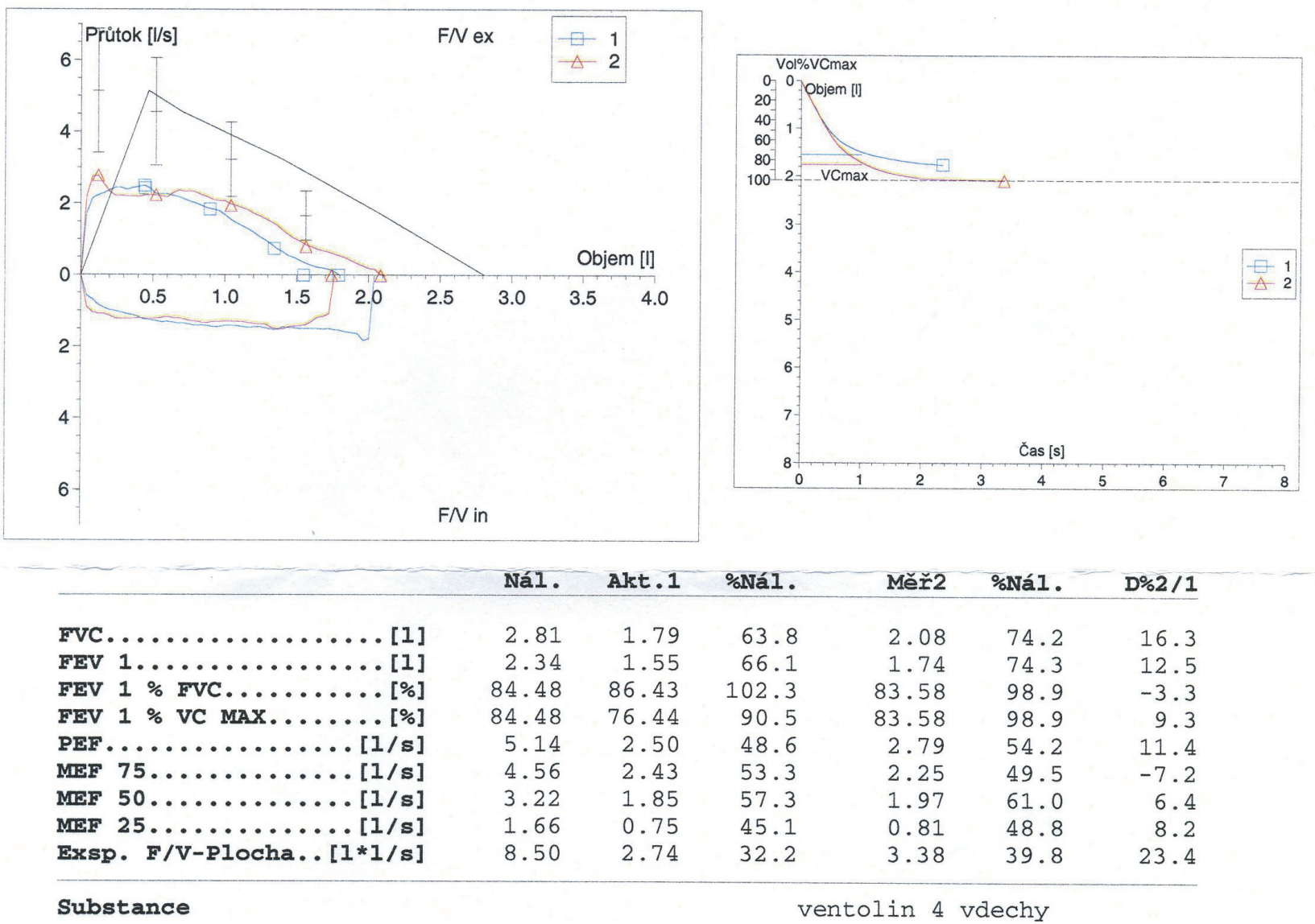

Fig. 3. Pulmonary function tests demonstrated reduced flow rates, with no post-bronchodilator improvement. 
A tracheal diverticulum was first described by Rokintansky in 1938. The frequency of tracheal diverticula found in some autopsy series has been estimated to be about $1 \%$; in children older than 10 years, it is reported as $0.3 \%$. A tracheal diverticulum is usually located approximately $4-5 \mathrm{~cm}$ below the vocal cords or just above the carina. Tracheal diverticula may be congenital or acquired. Congenital diverticula are not normally detected in infancy unless they are suggested by recurrent episodes of tracheobronchial infection or in association with other congenital malformations. The acquired form is thought to be due to prolonged increase in intraluminal pressure such as with a chronic cough. Although a tracheal diverticulum is usually asymptomatic, it may also accumulate respiratory secretions that become infected and lead to cough or tracheobronchitis ${ }^{1,2}$.

Bronchoscopy is usually needed to confirm the diagnosis of tracheal stenosis and a tracheal diverticulum. Computed tomography or magnetic resonance imaging may be useful to define the extent of narrowing. Advances in computed tomography technology with three-dimensional airway reconstruction have led to the development of virtual tracheobronchoscopy, which has enhanced the diagnosis of tracheobronchial anomalies.

Historically, laryngotracheal stenosis was managed with endoscopic antegrade bouginage as early as the 1970 s $^{3}$.

The successful use of balloon dilatation for benign airway stricture was first reported in an 18-week infant with post-surgical stricture. The mechanism of balloon dilatation of the airway relies on expanding the narrowed airway by creating longitudinal splits in the airway mucosa and tissues when the placed balloon is inflated by a pressure syringe device ${ }^{4}$.

Flexible bronchoscopic balloon dilatation is effective in some cases of benign fibrotic bronchial stenosis. The procedure is minimally invasive, safe, rapid and does not require general anesthesia. Modern balloons impart radially-directed force against the tracheal lumen, which is theoretically less traumatic to the laryngotracheal mucosa than the bougie. The balloon dilations are safe and efficient in the treatment of subglottic stenosis in children and reduce the need for open laryngeal surgery by $70 \%$ to $80 \%$. Good indications include primary or secondary treatment of acquired stenosis of all grades, secondary treatment of congenital stenosis, and primary treatment of some selected congenital cases ${ }^{5,6}$.

Mechanical dilatation of stenosis can be performed using rigid bronchoscopy and a series of metal bougies.

Nd:YAG laser photocoagulation has been used alone or with balloon dilatation. Laser resections are technically difficult and, when used for benign strictures, present a risk of bronchial perforation and/or bleeding ${ }^{7}$.
Surgical treatment of the stenotic segment is technically feasible in most patients but controversial due to the associated risks and a high rate of occurrence.

Airway stents generally are more effective than balloon dilatation when tracheo- or bronchomalacia is present.

Adjuvant therapies have included steroid injection, topical mitomycin C, and spray cryotherapy ${ }^{8}$.

\section{CONCLUSION}

The simultaneous occurrence of membranous tracheal stenosis and a tracheal diverticulum is rare and has not yet been described in the literature. The stenosis was vaporized with an Nd:YAG laser and dilated with a balloon. Due to recurrent stenosis, the laser procedure had to be repeated several times in approximately 4-6-month interval. The reason for membranous stenosis recurrence after the laser procedure is unknown to us. We are unable to explain either the origin of the stenosis, the tracheal diverticulum or the membranous stenosis recurrence.

\section{ABBREVIATIONS}

CT, computer tomography; TD, tracheal diverticulum.

Author contributions: Both authors contributed equally to preparing the manuscript.

Conflict of interest statement: The authors state that there are no conflicts of interest regarding the publication of this article.

\section{REFERENCES}

1. Ghaye B, Szapiro D, Fanchamps J-M, Dondelinger RF. Congenital bronchial abnormalities revisited. Radiographics 2001;21:105-19.

2. Restrepo S, Villamil MA, Rojas IC, Lemos DF, Echeverri S,Triana G, Angarita M. Association of two respiratory congenital anomalies: trachea diverticulum and cystic adenomatoiod malformation of the lung. Pediatr Radiol 2004;34:263-6.

3. Minnigerode B. Therapy of subglottic laryngeal stenosis in childhood using a hard rubber bougie, HNO 1972;20:370-1.

4. Cohen MD, Weber TR, Rao CC. Balloon dilatation of tracheal and bronchial stenosis. Am J Roentgenol 1984;142:477.

5. Hebra A, Powell DD, Smith CD, Othersen HB Jr. Balloon tracheoplasty in children: results of a 15-year experience, J Pediatr Surg 1991;26:957-61.

6. Chueng K, Chadha NK. Primary dilatation as a treatment for pediatric laryngotracheal stenosis: a systematic review. Int J Pediatr Otorhinolaryngol 2013;77(5):623-8.

7. Dumon JF, Reboud E, Garbe L, Aucomte F, Meric B. Treatment of tracheobronchial lesions by laser photoresection. Chest 1982;81:278.

8. Krimsky WS, Rodrigues MP, Malayaman N, Sarkar S. Spray cryotherapy for the treatment of glottic and subglottic stenosis, Laryngoscope 2010;120:473-7. 